\title{
Lax forms of the $q$-Painlevé equations
}

\author{
Mikio Murata \\ Department of Physics and Mathematics, \\ Aoyama Gakuin University, \\ 5-10-1 Fuchinobe Sagamihara-shi, \\ Kanagawa 229-8558, Japan
}

\begin{abstract}
All $q$-Painlevé equations which are obtained from the $q$-analog of the sixth Painlevé equation are expressed in a Lax formalism. They are characterized by the data of the associated linear $q$-difference equations. The degeneration pattern from the $q$-Painlevé equation of type $A_{2}$ is also presented.
\end{abstract}

Keywords: Painlevé equations, $q$-difference equations, Completely integrable systems.

2000 Mathematics Subject Classification: 33E17, 34M55, 39A12.

\section{Introduction}

Discrete Painlevé equations are studied from various points of view as integrable systems ([5]). They are discrete equations which are reduced to the Painlevé equations in suitable limiting process, and moreover, which pass the singularity confinement test. Passing this test can be thought of as a difference analogue of the Painlevé property. The singularity confinement test has been proposed by Grammaticos et al. as a criterion for the integrability of discrete dynamical systems ([2]).

Discrete Painlevé equations were classified on the basis of the types of rational surfaces connected to extended affine Weyl groups $([9,10])$. There are three types of discrete Painlevé equations: elliptic-difference, $q$-difference and difference. We especially give the list of $q$-Painlevé equations in the discrete Painlevé equations. See Table1. As is well-known, the sixth Painlevé equation yields the other five Painlevé equations by a process of coalescence. 


\begin{tabular}{|c||c|c|c|c|c|}
\hline Abbrev. & $q-P\left(A_{0}^{*}\right)$ & $q-P\left(A_{1}\right)$ & $q-P\left(A_{2}\right)$ & $q-P\left(A_{3}\right)$ & $q-P\left(A_{4}\right)$ \\
\hline Surface & $A_{0}^{(1) *}$ & $A_{1}^{(1)}$ & $A_{2}^{(1)}$ & $A_{3}^{(1)}$ & $A_{4}^{(1)}$ \\
\hline Symmetry & $E_{8}^{(1)}$ & $E_{7}^{(1)}$ & $E_{6}^{(1)}$ & $D_{5}^{(1)}$ & $A_{4}^{(1)}$ \\
\hline
\end{tabular}

\begin{tabular}{|c|c|c|c|c|c|}
\hline$q-P\left(A_{5}\right)$ & $q-P\left(A_{5}\right)^{\sharp}$ & $q-P\left(A_{6}\right)$ & $q-P\left(A_{6}\right)^{\sharp}$ & $q-P\left(A_{7}\right)$ & $q-P\left(A_{7}^{\prime}\right)$ \\
\hline$A_{5}^{(1)}$ & $A_{5}^{(1)}$ & $A_{6}^{(1)}$ & $A_{6}^{(1)}$ & $A_{7}^{(1)}$ & $A_{7}^{(1) \prime}$ \\
\hline$\left(A_{2}+A_{1}\right)^{(1)}$ & $\left(A_{2}+A_{1}\right)^{(1)}$ & $\left(A_{1}+A_{1}\right)^{(1)}$ & $\left(A_{1}+A_{1}\right)^{(1)}$ & $A_{1}^{(1)}$ & $A_{1}^{(1)}$ \\
\hline
\end{tabular}

Table 1: The q-Painlevé equations

Among the $q$-Painlevé equations, the $q$-Painlevé equation of type $A_{0}^{*}(q-$ $\left.P\left(A_{0}^{*}\right)\right)$ is the most generic one because the other $q$-Painlevé equations can be obtained from this equation by limiting procedure. These equations are organized in a degeneration pattern obtained through coalescence. See Table 2.

$$
\begin{aligned}
& q-P\left(A_{0}^{*}\right) \quad \rightarrow \quad q-P\left(A_{1}\right) \quad \rightarrow \quad q-P\left(A_{2}\right) \quad \rightarrow \quad q-P\left(A_{3}\right) \quad \rightarrow
\end{aligned}
$$

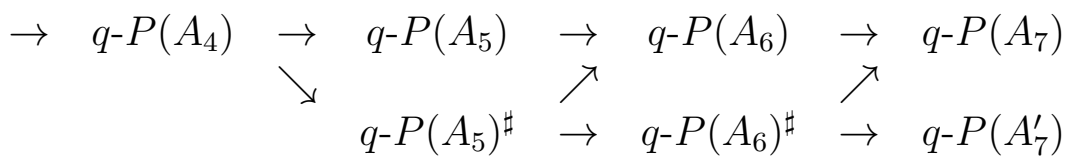

Table 2: Degeneration pattern for the $q$-Painlevé equations

Another important aspect of the Painlevé equations is their connection to the monodromy-preserving deformation of linear differential equations. The generalized Riemann problem was already studied for linear differential, difference and $q$-difference equations in the Birkhoff's paper, [1]. Jimbo and Sakai studied the deformation of a $2 \times 2$ matrix system of $q$-difference equations and found the $q$-Painlevé equation of type $A_{3}\left(q-P\left(A_{3}\right)\right)$, which is commonly known as $q-\mathrm{P}_{\mathrm{VI}}([4])$. Sakai also found a Lax form of the $q$ Painlevé equation of type $A_{2}\left(q-P\left(A_{2}\right)\right)$, a particular case of a $q$-Garnier system $([7,8])$. Hay et al. found Lax forms of $q$-Painlevé equations, reductions from a Lax pair for a lattice modified $\mathrm{KdV}$ equation ([3]). However, Lax forms of a lot of $q$-Painlevé equations have not been obtained yet.

In this paper, we present Lax pairs of all $q$-Painlevé equations which are obtained from $q-P\left(A_{3}\right)$. In Section 2 , we illustrate the connection preserving deformation and derive $q-P\left(A_{3}\right)$. We also propose Lax pairs of $q-P\left(A_{4}\right)$, q$P\left(A_{5}\right), q-P\left(A_{5}\right)^{\sharp}, q-P\left(A_{6}\right), q-P\left(A_{6}\right)^{\sharp}, q-P\left(A_{7}\right)$ and $q-P\left(A_{7}^{\prime}\right)$. In Section 3 , we give replacements of the parameters for the degeneration. The Lax form of $q-P\left(A_{3}\right)$ can be obtained from the Lax form of $q-P\left(A_{2}\right)$. In Section 4 , we give the Lax form of $q-P\left(A_{2}\right)$ and replacements of the parameters for the 
degeneration.

\section{Lax forms of q-Painlevé equations}

\subsection{Derivation of $q-P\left(A_{3}\right)$}

In this section, we illustrate the connection preserving deformation and derive the $q$-Painlevé equation of type $A_{3}$.

Consider a $2 \times 2$ matrix system with polynomial coefficients

$$
Y(q x, t)=A(x, t) Y(x, t) .
$$

The connection preserving deformation of the linear $q$-difference equation, which is a discrete counterpart of monodromy preserving deformation, is equivalent to existence of a linear deformation equation whose coefficients are rational in $x$. We express the deformation equation in the form

$$
Y(x, q t)=B(x, t) Y(x, t) .
$$

The compatibility condition for the systems (2.1) and (2.2) reads

$$
A(x, q t) B(x, t)=B(q x, t) A(x, t) .
$$

$q-P\left(A_{3}\right)$ can be obtained from the condition (2.3).

We take $A(x, t)$ to be of the form

$$
\begin{gathered}
A(x, t)=A_{0}(t)+x A_{1}(t)+x^{2} A_{2}, \\
A_{2}=\left(\begin{array}{cc}
\kappa_{1} & 0 \\
0 & \kappa_{2}
\end{array}\right), \quad A_{0}(t) \text { has eigenvalues } \theta_{1} t, \theta_{2} t, \\
\operatorname{det} A(x, t)=\kappa_{1} \kappa_{2}\left(x-a_{1} t\right)\left(x-a_{2} t\right)\left(x-a_{3}\right)\left(x-a_{4}\right) .
\end{gathered}
$$

Here the parameters $\kappa_{j}, \theta_{j}, a_{j}$ are independent of $t$. We have

$$
\kappa_{1} \kappa_{2} a_{1} a_{2} a_{3} a_{4}=\theta_{1} \theta_{2} .
$$

Define $y=y(t), z_{i}=z_{i}(t)(i=1,2)$ by

$$
A_{12}(y, t)=0, \quad A_{11}(y, t)=\kappa_{1} z_{1}, \quad A_{22}(y, t)=\kappa_{2} z_{2},
$$

so that

$$
z_{1} z_{2}=\kappa_{1} \kappa_{2}\left(y-a_{1} t\right)\left(y-a_{2} t\right)\left(y-a_{3}\right)\left(y-a_{4}\right) .
$$


The matrix $A(x, t)$ can be parametrized as

$$
A(x, t)=\left(\begin{array}{cc}
\kappa_{1}\left((x-y)(x-\alpha)+z_{1}\right) & \kappa_{2} w(x-y) \\
\kappa_{1} w^{-1}(\gamma x+\delta) & \kappa_{2}\left((x-y)(x-\beta)+z_{2}\right)
\end{array}\right) .
$$

Here

$$
\begin{aligned}
\alpha= & \frac{1}{\kappa_{1}-\kappa_{2}}\left[y^{-1}\left(\left(\theta_{1}+\theta_{2}\right) t-\kappa_{1} z_{1}-\kappa_{2} z_{2}\right)\right. \\
& \left.-\kappa_{2}\left(\left(a_{1}+a_{2}\right) t+a_{3}+a_{4}-2 y\right)\right], \\
\beta= & \frac{1}{\kappa_{1}-\kappa_{2}}\left[-y^{-1}\left(\left(\theta_{1}+\theta_{2}\right) t-\kappa_{1} z_{1}-\kappa_{2} z_{2}\right)\right. \\
& \left.+\kappa_{1}\left(\left(a_{1}+a_{2}\right) t+a_{3}+a_{4}-2 y\right)\right], \\
\gamma= & z_{1}+z_{2}+(y+\alpha)(y+\beta)+(\alpha+\beta) y-a_{1} a_{2} t^{2} \\
& -\left(a_{1}+a_{2}\right)\left(a_{3}+a_{4}\right) t-a_{3} a_{4}, \\
\delta= & y^{-1}\left(a_{1} a_{2} a_{3} a_{4} t^{2}-\left(\alpha y+z_{1}\right)\left(\beta y+z_{2}\right)\right) .
\end{aligned}
$$

The quantity $w=w(t)$ is related to the 'gauge' freedom, and does not enter the final result for the $q-P\left(A_{3}\right)$. The matrix $B(x, t)$ is a rational function of the form

$$
B(x, t)=\frac{x}{\left(x-a_{1} q t\right)\left(x-a_{2} q t\right)}\left(x I+B_{0}(t)\right) .
$$

The compatibility (2.3) is equivalent to

$$
\begin{gathered}
A\left(a_{i} q t, q t\right)\left(a_{i} q t I+B_{0}(t)\right)=0 \quad(i=1,2), \\
\left(a_{i} q t I+B_{0}(t)\right) A\left(a_{i} t, t\right)=0 \quad(i=1,2), \\
A_{0}(q t) B_{0}(t)=q B_{0}(t) A_{0}(t) .
\end{gathered}
$$

Substituting the parametrization above, one obtains a set of $q$-difference equations. Let us use the notations $\bar{y}=y(q t)$ and so forth. Introduce $z$ by

$$
z_{1}=\frac{\left(y-a_{1} t\right)\left(y-a_{2} t\right)}{\kappa_{1} q z}, \quad z_{2}=\kappa_{1} q z\left(y-a_{3}\right)\left(y-a_{4}\right) .
$$


Then the matrix $B_{0}(t)=\left(B_{i j}\right)$ is parametrized as follows:

$$
\begin{aligned}
B_{11} & =\frac{-\kappa_{2} q \bar{z}}{1-\kappa_{2} \bar{z}}\left(-\beta+\frac{t\left(a_{1}+a_{2}\right)-y}{\kappa_{2} \bar{z}}\right), \\
B_{12} & =\frac{\kappa_{2} q w \bar{z}}{1-\kappa_{2} \bar{z}}, \\
B_{21} & =\frac{\kappa_{1} q \bar{z}}{w\left(1-\kappa_{1} q \bar{z}\right)}\left(a_{1} q t-\bar{\alpha}+\frac{a_{2} q t-\bar{y}}{\kappa_{1} q \bar{z}}\right)\left(a_{1} t-\beta+\frac{a_{2} t-y}{\kappa_{2} \bar{z}}\right) \\
& =\frac{\kappa_{1} q \bar{z}}{w\left(1-\kappa_{1} q \bar{z}\right)}\left(a_{2} q t-\bar{\alpha}+\frac{a_{1} q t-\bar{y}}{\kappa_{1} q \bar{z}}\right)\left(a_{2} t-\beta+\frac{a_{1} t-y}{\kappa_{2} \bar{z}}\right), \\
B_{22} & =\frac{-\kappa_{1} q \bar{z}}{1-\kappa_{1} q \bar{z}}\left(-\bar{\alpha}+\frac{q t\left(a_{1}+a_{2}\right)-\bar{y}}{\kappa_{1} q \bar{z}}\right) .
\end{aligned}
$$

Set further

$$
b_{1}=\frac{a_{1} a_{2}}{\theta_{1}}, \quad b_{2}=\frac{a_{1} a_{2}}{\theta_{2}}, \quad b_{3}=\frac{1}{\kappa_{1} q}, \quad b_{4}=\frac{1}{\kappa_{2}} .
$$

Equations (2.16) $-(2.18)$ are equivalent to

$$
\begin{aligned}
\frac{y \bar{y}}{a_{3} a_{4}} & =\frac{\left(\bar{z}-b_{1} t\right)\left(\bar{z}-b_{2} t\right)}{\left(\bar{z}-b_{3}\right)\left(\bar{z}-b_{4}\right)} \\
\frac{z \bar{z}}{b_{3} b_{4}} & =\frac{\left(y-a_{1} t\right)\left(y-a_{2} t\right)}{\left(y-a_{3}\right)\left(y-a_{4}\right)} \\
\frac{\bar{w}}{w} & =\frac{b_{4}}{b_{3}} \frac{\bar{z}-b_{3}}{\bar{z}-b_{4}} .
\end{aligned}
$$

We have a single constraint

$$
\frac{b_{1} b_{2}}{b_{3} b_{4}}=q \frac{a_{1} a_{2}}{a_{3} a_{4}}
$$

$q-P\left(A_{3}\right)$ is (2.21) and (2.22).

\subsection{Lax form of $q-P\left(A_{4}\right)$}

Consider a $2 \times 2$ matrix system with polynomial coefficients

$$
Y(q x, t)=A(x, t) Y(x, t) .
$$

We express the deformation equation in the form

$$
Y(x, q t)=B(x, t) Y(x, t)
$$


and can express the $q$-Painlevé equation of type $A_{4}$ in the form

$$
A(x, q t) B(x, t)=B(q x, t) A(x, t)
$$

by the compatibility of the deformation equation and the original linear $q$ difference equation.

We take $A(x, t)$ to be of the form

$$
\begin{gathered}
A(x, t)=A_{0}(t)+x A_{1}(t)+x^{2} A_{2}, \\
A_{2}=\left(\begin{array}{cc}
\kappa_{1} & 0 \\
0 & 0
\end{array}\right), \quad A_{0}(t) \text { has eigenvalues } \theta_{1} t, \theta_{2} t, \\
\operatorname{det} A(x, t)=\kappa_{1} \kappa_{2}\left(x-a_{1} t\right)\left(x-a_{2} t\right)\left(x-a_{3}\right) .
\end{gathered}
$$

We have

$$
-\kappa_{1} \kappa_{2} a_{1} a_{2} a_{3}=\theta_{1} \theta_{2} .
$$

The matrix $B(x, t)$ is a rational function of the form

$$
B(x, t)=\frac{x}{\left(x-a_{1} q t\right)\left(x-a_{2} q t\right)}\left(x I+B_{0}(t)\right) .
$$

Define $y=y(t), z_{i}=z_{i}(t)(i=1,2)$ by

$$
A_{12}(y, t)=0, \quad A_{11}(y, t)=\kappa_{1} z_{1}, \quad A_{22}(y, t)=z_{2},
$$

so that

$$
z_{1} z_{2}=\kappa_{2}\left(y-a_{1} t\right)\left(y-a_{2} t\right)\left(y-a_{3}\right) .
$$

The matrix $A(x, t)$ can be parametrized as

$$
A(x, t)=\left(\begin{array}{cc}
\kappa_{1}\left((x-y)(x-\alpha)+z_{1}\right) & w(x-y) \\
\kappa_{1} w^{-1}(\gamma x+\delta) & \kappa_{2}(x-y)+z_{2}
\end{array}\right) .
$$

Here

$$
\begin{aligned}
\alpha & =\frac{1}{\kappa_{1}}\left[y^{-1}\left(\left(\theta_{1}+\theta_{2}\right) t-\kappa_{1} z_{1}-z_{2}\right)+\kappa_{2}\right], \\
\gamma & =z_{2}-\kappa_{2}\left((2 y+\alpha)-\left(a_{1}+a_{2}\right) t-a_{3}\right), \\
\delta & =y^{-1}\left(-\kappa_{2} a_{1} a_{2} a_{3} t^{2}-\left(\alpha y+z_{1}\right)\left(-\kappa_{2} y+z_{2}\right)\right) .
\end{aligned}
$$

Introduce $z$ by

$$
z_{1}=\frac{\left(y-a_{1} t\right)\left(y-a_{2} t\right)}{\kappa_{1} q z}, \quad z_{2}=\kappa_{1} \kappa_{2} q z\left(y-a_{3}\right) .
$$


Then the matrix $B_{0}(t)=\left(B_{i j}\right)$ is parametrized as follows:

$$
\begin{aligned}
B_{11} & =-q \bar{z}\left(\kappa_{2}+\frac{t\left(a_{1}+a_{2}\right)-y}{\bar{z}}\right) \\
B_{12} & =q w \bar{z} \\
B_{21} & =\frac{\kappa_{1} q \bar{z}}{w\left(1-\kappa_{1} q \bar{z}\right)}\left(a_{1} q t-\bar{\alpha}+\frac{a_{2} q t-\bar{y}}{\kappa_{1} q \bar{z}}\right)\left(\kappa_{2}+\frac{a_{2} t-y}{\bar{z}}\right) \\
& =\frac{\kappa_{1} q \bar{z}}{w\left(1-\kappa_{1} q \bar{z}\right)}\left(a_{2} q t-\bar{\alpha}+\frac{a_{1} q t-\bar{y}}{\kappa_{1} q \bar{z}}\right)\left(\kappa_{2}+\frac{a_{1} t-y}{\bar{z}}\right) \\
B_{22} & =\frac{-\kappa_{1} q \bar{z}}{1-\kappa_{1} q \bar{z}}\left(-\bar{\alpha}+\frac{q t\left(a_{1}+a_{2}\right)-\bar{y}}{\kappa_{1} q \bar{z}}\right) .
\end{aligned}
$$

Set further

$$
b_{1}=\frac{a_{1} a_{2}}{\theta_{1}}, \quad b_{2}=\frac{a_{1} a_{2}}{\theta_{2}}, \quad b_{3}=\frac{1}{\kappa_{1} q}, \quad a_{4}=-\kappa_{2} .
$$

Equation (2.27) are equivalent to

$$
\begin{aligned}
\frac{y \bar{y}}{a_{3} a_{4}} & =-\frac{\left(\bar{z}-b_{1} t\right)\left(\bar{z}-b_{2} t\right)}{\bar{z}-b_{3}}, \\
\frac{z \bar{z}}{b_{3}} & =-\frac{\left(y-a_{1} t\right)\left(y-a_{2} t\right)}{a_{4}\left(y-a_{3}\right)}, \\
\frac{\bar{w}}{w} & =-\frac{\bar{z}}{b_{3}}+1 .
\end{aligned}
$$

We have a constraint

$$
\frac{b_{1} b_{2}}{b_{3}}=q \frac{a_{1} a_{2}}{a_{3} a_{4}}
$$

$q-P\left(A_{4}\right)$ is (2.45) and (2.46).

\subsection{Lax form of $q-P\left(A_{5}\right)$}

Consider a $2 \times 2$ matrix system with polynomial coefficients

$$
Y(q x, t)=A(x, t) Y(x, t) .
$$

We express the deformation equation

$$
Y(x, q t)=B(x, t) Y(x, t)
$$

and can express the $q$-Painlevé equation of type $A_{5}$ in the form

$$
A(x, q t) B(x, t)=B(q x, t) A(x, t)
$$


by the compatibility of the deformation equation and the original linear $q$ difference equation.

We take $A(x, t)$ to be of the form

$$
\begin{gathered}
A(x, t)=A_{0}(t)+x A_{1}(t)+x^{2} A_{2}, \\
A_{2}=\left(\begin{array}{cc}
\kappa_{1} & 0 \\
0 & 0
\end{array}\right), \quad A_{0}(t) \text { has eigenvalues } \theta_{1} t, 0, \\
\operatorname{det} A(x, t)=\kappa_{1} \kappa_{2} x\left(x-a_{1} t\right)\left(x-a_{2} t\right) .
\end{gathered}
$$

The matrix $B(x, t)$ is a rational function of the form

$$
B(x, t)=\frac{x}{\left(x-a_{1} q t\right)\left(x-a_{2} q t\right)}\left(x I+B_{0}(t)\right) .
$$

Define $y=y(t), z_{i}=z_{i}(t)(i=1,2)$ by

$$
A_{12}(y, t)=0, \quad A_{11}(y, t)=\kappa_{1} z_{1}, \quad A_{22}(y, t)=z_{2},
$$

so that

$$
z_{1} z_{2}=\kappa_{2} y\left(y-a_{1} t\right)\left(y-a_{2} t\right) .
$$

The matrix $A(x, t)$ can be parametrized as

$$
A(x, t)=\left(\begin{array}{cc}
\kappa_{1}\left((x-y)(x-\alpha)+z_{1}\right) & w(x-y) \\
\kappa_{1} w^{-1}(\gamma x+\delta) & \kappa_{2}(x-y)+z_{2}
\end{array}\right) .
$$

Here

$$
\begin{aligned}
\alpha & =\frac{1}{\kappa_{1}}\left[y^{-1}\left(\theta_{1} t-\kappa_{1} z_{1}-z_{2}\right)+\kappa_{2}\right], \\
\gamma & =z_{2}-\kappa_{2}\left(2 y+\alpha-t\left(a_{1}+a_{2}\right)\right), \\
\delta & =-y^{-1}\left(\alpha y+z_{1}\right)\left(-\kappa_{2} y+z_{2}\right) .
\end{aligned}
$$

Introduce $z$ by

$$
z_{1}=\frac{\left(y-a_{1} t\right)\left(y-a_{2} t\right)}{\kappa_{1} q z}, \quad z_{2}=\kappa_{1} \kappa_{2} q z y
$$


Then the matrix $B_{0}(t)=\left(B_{i j}\right)$ is parametrized as follows:

$$
\begin{aligned}
B_{11} & =-q \bar{z}\left(\kappa_{2}+\frac{t\left(a_{1}+a_{2}\right)-y}{\bar{z}}\right) \\
B_{12} & =q w \bar{z} \\
B_{21} & =\frac{\kappa_{1} q \bar{z}}{w\left(1-\kappa_{1} q \bar{z}\right)}\left(a_{1} q t-\bar{\alpha}+\frac{a_{2} q t-\bar{y}}{\kappa_{1} q \bar{z}}\right)\left(\kappa_{2}+\frac{a_{2} t-y}{\bar{z}}\right) \\
& =\frac{\kappa_{1} q \bar{z}}{w\left(1-\kappa_{1} q \bar{z}\right)}\left(a_{2} q t-\bar{\alpha}+\frac{a_{1} q t-\bar{y}}{\kappa_{1} q \bar{z}}\right)\left(\kappa_{2}+\frac{a_{1} t-y}{\bar{z}}\right) \\
B_{22} & =\frac{-\kappa_{1} q \bar{z}}{1-\kappa_{1} q \bar{z}}\left(-\bar{\alpha}+\frac{q t\left(a_{1}+a_{2}\right)-\bar{y}}{\kappa_{1} q \bar{z}}\right) .
\end{aligned}
$$

Set further

$$
b_{1}=\frac{a_{1} a_{2}}{\theta_{1}}, \quad b_{2}=-\frac{\theta_{1}}{\kappa_{1} \kappa_{2}}, \quad b_{3}=\frac{1}{\kappa_{1} q}, \quad a_{4}=-\kappa_{2} .
$$

Equation (2.51) are equivalent to

$$
\begin{aligned}
\frac{y \bar{y}}{a_{3} a_{4}} & =\frac{b_{2} t\left(\bar{z}-b_{1} t\right)}{\bar{z}-b_{3}}, \\
\frac{z \bar{z}}{b_{3}} & =-\frac{\left(y-a_{1} t\right)\left(y-a_{2} t\right)}{a_{4} y}, \\
\frac{\bar{w}}{w} & =-\frac{\bar{z}}{b_{3}}+1 .
\end{aligned}
$$

We have a constraint

$$
\frac{b_{1} b_{2}}{b_{3}}=q \frac{a_{1} a_{2}}{a_{4}}
$$

$q-P\left(A_{5}\right)$ is (2.68) and (2.69).

\subsection{Lax form of $q-P\left(A_{5}\right)^{\#}$}

Consider a $2 \times 2$ matrix system with polynomial coefficients

$$
Y(q x, t)=A(x, t) Y(x, t) .
$$

We express the deformation equation

$$
Y(x, q t)=B(x, t) Y(x, t)
$$

and can express the $q$-Painlevé equation of type $A_{5}^{\sharp}$ in the form

$$
A(x, q t) B(x, t)=B(q x, t) A(x, t)
$$


by the compatibility of the deformation equation and the original linear $q$ difference equation.

We take $A(x, t)$ to be of the form

$$
\begin{gathered}
A(x, t)=A_{0}(t)+x A_{1}(t)+x^{2} A_{2}, \\
A_{2}=\left(\begin{array}{cc}
\kappa_{1} & 0 \\
0 & 0
\end{array}\right), \quad A_{0}(t) \text { has eigenvalues } \theta_{1} t, 0, \\
\operatorname{det} A(x, t)=\kappa_{1} \kappa_{2} x\left(x-a_{1} t\right)\left(x-a_{3}\right) .
\end{gathered}
$$

The matrix $B(x, t)$ is a rational function of the form

$$
B(x, t)=\frac{1}{x-a_{1} q t}\left(x I+B_{0}(t)\right)
$$

Define $y=y(t), z_{i}=z_{i}(t)(i=1,2)$ by

$$
A_{12}(y, t)=0, \quad A_{11}(y, t)=\kappa_{1} z_{1}, \quad A_{22}(y, t)=z_{2},
$$

so that

$$
z_{1} z_{2}=\kappa_{2} y\left(y-a_{1} t\right)\left(y-a_{3}\right) .
$$

The matrix $A(x, t)$ can be parametrized as

$$
A(x, t)=\left(\begin{array}{cc}
\kappa_{1}\left((x-y)(x-\alpha)+z_{1}\right) & w(x-y) \\
\kappa_{1} w^{-1}(\gamma x+\delta) & \kappa_{2}(x-y)+z_{2}
\end{array}\right) .
$$

Here

$$
\begin{aligned}
\alpha & =\frac{1}{\kappa_{1}}\left[y^{-1}\left(\theta_{1} t-\kappa_{1} z_{1}-z_{2}\right)+\kappa_{2}\right], \\
\gamma & =z_{2}-\kappa_{2}\left(2 y+\alpha-a_{1} t-a_{3}\right), \\
\delta & =-y^{-1}\left(\alpha y+z_{1}\right)\left(-\kappa_{2} y+z_{2}\right) .
\end{aligned}
$$

Introduce $z$ by

$$
z_{1}=\frac{y\left(y-a_{1} t\right)}{\kappa_{1} q z}, \quad z_{2}=\kappa_{1} \kappa_{2} q z\left(y-a_{3}\right)
$$


Then the matrix $B_{0}(t)=\left(B_{i j}\right)$ is parametrized as follows:

$$
\begin{aligned}
B_{11} & =-q \bar{z}\left(\kappa_{2}+\frac{a_{1} t-y}{\bar{z}}\right) \\
B_{12} & =q w \bar{z} \\
B_{21} & =\frac{\kappa_{1} q \bar{z}}{w\left(1-\kappa_{1} q \bar{z}\right)}\left(a_{1} q t-\bar{\alpha}-\frac{\bar{y}}{\kappa_{1} q \bar{z}}\right)\left(\kappa_{2}-\frac{y}{\bar{z}}\right) \\
& =\frac{\kappa_{1} q \bar{z}}{w\left(1-\kappa_{1} q \bar{z}\right)}\left(-\bar{\alpha}+\frac{a_{1} q t-\bar{y}}{\kappa_{1} q \bar{z}}\right)\left(\kappa_{2}+\frac{a_{1} t-y}{\bar{z}}\right), \\
B_{22} & =\frac{-\kappa_{1} q \bar{z}}{1-\kappa_{1} q \bar{z}}\left(-\bar{\alpha}+\frac{a_{1} q t-\bar{y}}{\kappa_{1} q \bar{z}}\right) .
\end{aligned}
$$

Set further

$$
b_{1}=\frac{a_{1}}{\theta_{1}}, \quad b_{2}=-\frac{\theta_{1}}{\kappa_{1} \kappa_{2} a_{3}}, \quad b_{3}=\frac{1}{\kappa_{1} q}, \quad a_{4}=-\kappa_{2} .
$$

Equation (2.74) are equivalent to

$$
\begin{aligned}
\frac{y \bar{y}}{a_{3} a_{4}} & =-\frac{\bar{z}\left(\bar{z}-b_{2} t\right)}{\bar{z}-b_{3}}, \\
\frac{z \bar{z}}{b_{3}} & =-\frac{y\left(y-a_{1} t\right)}{a_{4}\left(y-a_{3}\right)}, \\
\frac{\bar{w}}{w} & =-\frac{\bar{z}}{b_{3}}+1 .
\end{aligned}
$$

We have a constraint

$$
\frac{b_{1} b_{2}}{b_{3}}=q \frac{a_{1}}{a_{3} a_{4}}
$$

$q-P\left(A_{5}\right)^{\sharp}$ is (2.91) and (2.92).

\subsection{Lax form of $q-P\left(A_{6}\right)$}

Consider a $2 \times 2$ matrix system with polynomial coefficients

$$
Y(q x, t)=A(x, t) Y(x, t) .
$$

We express the deformation equation

$$
Y(x, q t)=B(x, t) Y(x, t)
$$

and can express the $q$-Painlevé equation type $A_{6}$ in the form

$$
A(x, q t) B(x, t)=B(q x, t) A(x, t)
$$


by the compatibility of the deformation equation and the original linear $q$ difference equation.

We take $A(x, t)$ to be of the form

$$
\begin{gathered}
A(x, t)=A_{0}(t)+x A_{1}(t)+x^{2} A_{2}, \\
A_{2}=\left(\begin{array}{cc}
\kappa_{1} & 0 \\
0 & 0
\end{array}\right), \quad A_{0}(t) \text { has eigenvalues } \theta_{1} t, 0, \\
\operatorname{det} A(x, t)=\kappa_{1} \kappa_{2} x^{2}\left(x-a_{1} t\right) .
\end{gathered}
$$

The matrix $B(x, t)$ is a rational function of the form

$$
B(x, t)=\frac{1}{x-a_{1} q t}\left(x I+B_{0}(t)\right) .
$$

Define $y=y(t), z_{i}=z_{i}(t)(i=1,2)$ by

$$
A_{12}(y, t)=0, \quad A_{11}(y, t)=\kappa_{1} z_{1}, \quad A_{22}(y, t)=z_{2},
$$

so that

$$
z_{1} z_{2}=\kappa_{2} y^{2}\left(y-a_{1} t\right) .
$$

The matrix $A(x, t)$ can be parametrized as

$$
A(x, t)=\left(\begin{array}{cc}
\kappa_{1}\left((x-y)(x-\alpha)+z_{1}\right) & w(x-y) \\
\kappa_{1} w^{-1}(\gamma x+\delta) & \kappa_{2}(x-y)+z_{2}
\end{array}\right) .
$$

Here

$$
\begin{aligned}
\alpha & =\frac{1}{\kappa_{1}}\left[y^{-1}\left(\theta_{1} t-\kappa_{1} z_{1}-z_{2}\right)+\kappa_{2}\right] \\
\gamma & =z_{2}-\kappa_{2}\left(2 y+\alpha-a_{1} t\right) \\
\delta & =-y^{-1}\left(\alpha y+z_{1}\right)\left(-\kappa_{2} y+z_{2}\right) .
\end{aligned}
$$

Introduce $z$ by

$$
z_{1}=\frac{y\left(y-a_{1} t\right)}{\kappa_{1} q z}, \quad z_{2}=\kappa_{1} \kappa_{2} q z y .
$$

Then the matrix $B_{0}(t)=\left(B_{i j}\right)$ is parametrized as follows:

$$
\begin{aligned}
B_{11} & =-q \bar{z}\left(\kappa_{2}+\frac{a_{1} t-y}{\bar{z}}\right), \\
B_{12} & =q w \bar{z}, \\
B_{21} & =\frac{\kappa_{1} q \bar{z}}{w\left(1-\kappa_{1} q \bar{z}\right)}\left(a_{1} q t-\bar{\alpha}-\frac{\bar{y}}{\kappa_{1} q \bar{z}}\right)\left(\kappa_{2}-\frac{y}{\bar{z}}\right) \\
& =\frac{\kappa_{1} q \bar{z}}{w\left(1-\kappa_{1} q \bar{z}\right)}\left(-\bar{\alpha}+\frac{a_{1} q t-\bar{y}}{\kappa_{1} q \bar{z}}\right)\left(\kappa_{2}+\frac{a_{1} t-y}{\bar{z}}\right) . \\
B_{22} & =\frac{-\kappa_{1} q \bar{z}}{1-\kappa_{1} q \bar{z}}\left(-\bar{\alpha}+\frac{a_{1} q t-\bar{y}}{\kappa_{1} q \bar{z}}\right) .
\end{aligned}
$$


Set further

$$
b_{1}=\frac{a_{1}}{\theta_{1}}, \quad b_{2}=-\frac{\theta_{1}}{\kappa_{1} \kappa_{2}}, \quad b_{3}=\frac{1}{\kappa_{1} q}, \quad b_{4}=-\kappa_{2} .
$$

Equation (2.97) are equivalent to

$$
\begin{aligned}
\frac{y \bar{y}}{a_{4}} & =\frac{b_{2} t \bar{z}}{\bar{z}-b_{3}}, \\
\frac{z \bar{z}}{b_{3}} & =-\frac{y\left(y-a_{1} t\right)}{a_{4} y}, \\
\frac{\bar{w}}{w} & =-\frac{\bar{z}}{b_{3}}+1 .
\end{aligned}
$$

We have a constraint

$$
\frac{b_{1} b_{2}}{b_{3}}=q \frac{a_{1}}{a_{4}} .
$$

$q-P\left(A_{6}\right)$ is (2.114) and (2.115).

\subsection{Lax form of $q-P\left(A_{6}\right)^{\sharp}$}

Consider a $2 \times 2$ matrix system with polynomial coefficients

$$
Y(q x, t)=A(x, t) Y(x, t) .
$$

We express the deformation equation

$$
Y(x, q t)=B(x, t) Y(x, t)
$$

and can express the $q$-Painlevé equation of type $A_{6}{ }^{\sharp}$ in the form

$$
A(x, q t) B(x, t)=B(q x, t) A(x, t)
$$

by the compatibility of the deformation equation and the original linear $q$ difference equation.

We take $A(x, t)$ to be of the form

$$
\begin{gathered}
A(x, t)=A_{0}(t)+x A_{1}(t)+x^{2} A_{2}, \\
A_{2}=\left(\begin{array}{cc}
\kappa_{1} & 0 \\
0 & 0
\end{array}\right), \quad A_{0}(t) \text { has eigenvalues } \theta_{1} t, 0, \\
\operatorname{det} A(x, t)=\kappa_{1} \kappa_{2} x^{2}\left(x-a_{3}\right),
\end{gathered}
$$

The matrix $B(x, t)$ is a rational function of the form

$$
B(x, t)=\frac{1}{x}\left(x I+B_{0}(t)\right) .
$$


Define $y=y(t), z_{i}=z_{i}(t)(i=1,2)$ by

$$
A_{12}(y, t)=0, \quad A_{11}(y, t)=\kappa_{1} z_{1}, \quad A_{22}(y, t)=z_{2},
$$

so that

$$
z_{1} z_{2}=\kappa_{2} y^{2}\left(y-a_{3}\right) .
$$

The matrix $A(x, t)$ can be parametrized as

$$
A(x, t)=\left(\begin{array}{cc}
\kappa_{1}\left((x-y)(x-\alpha)+z_{1}\right) & w(x-y) \\
\kappa_{1} w^{-1}(\gamma x+\delta) & \kappa_{2}(x-y)+z_{2}
\end{array}\right) .
$$

Here

$$
\begin{aligned}
\alpha & =\frac{1}{\kappa_{1}}\left[y^{-1}\left(\theta_{1} t-\kappa_{1} z_{1}-z_{2}\right)+\kappa_{2}\right], \\
\gamma & =z_{2}-\kappa_{2}\left(2 y+\alpha-a_{3}\right) \\
\delta & =-y^{-1}\left(\alpha y+z_{1}\right)\left(-\kappa_{2} y+z_{2}\right) .
\end{aligned}
$$

Introduce $z$ by

$$
z_{1}=\frac{y^{2}}{\kappa_{1} q z}, \quad z_{2}=\kappa_{1} \kappa_{2} q z\left(y-a_{3}\right) .
$$

Then the matrix $B_{0}(t)=\left(B_{i j}\right)$ is parametrized as follows:

$$
\begin{aligned}
B_{11} & =-q \bar{z}\left(\kappa_{2}-\frac{y}{\kappa_{2} \bar{z}}\right), \\
B_{12} & =q w \bar{z} \\
B_{21} & =\frac{\kappa_{1} q}{w\left(1-\kappa_{1} q \bar{z}\right)}\left(-\bar{\alpha}-\frac{\bar{y}}{\kappa_{1} q \bar{z}}\right)\left(\kappa_{2}-\frac{y}{\bar{z}}\right), \\
B_{22} & =\frac{-\kappa_{1} q \bar{z}}{1-\kappa_{1} q \bar{z}}\left(-\bar{\alpha}-\frac{\bar{y}}{\kappa_{1} q \bar{z}}\right) .
\end{aligned}
$$

Set further

$$
b_{1}=\frac{1}{\theta_{1}}, \quad b_{2}=-\frac{\theta_{1}}{\kappa_{1} \kappa_{2} a_{3}}, \quad b_{3}=\frac{1}{\kappa_{1} q}, \quad a_{4}=-\kappa_{2} .
$$

Equation (2.120) are equivalent to

$$
\begin{aligned}
\frac{y \bar{y}}{a_{3} a_{4}} & =-\frac{\bar{z}\left(\bar{z}-b_{2} t\right)}{\bar{z}-b_{3}}, \\
\frac{z \bar{z}}{b_{3}} & =-\frac{y^{2}}{a_{4}\left(y-a_{3}\right)}, \\
\frac{\bar{w}}{w} & =-\frac{\bar{z}}{b_{3}}+1 .
\end{aligned}
$$


We have a constraint

$$
\frac{b_{1} b_{2}}{b_{3}}=q \frac{1}{a_{3} a_{4}} .
$$

$q-P\left(A_{6}\right)^{\sharp}$ is (2.137) and (2.138).

\subsection{Lax form of $q-P\left(A_{7}\right)$}

Consider a $2 \times 2$ matrix system with polynomial coefficients

$$
Y(q x, t)=A(x, t) Y(x, t) .
$$

We express the deformation equation

$$
Y(x, q t)=B(x, t) Y(x, t)
$$

and can express the $q$-Painlevé equation of type $A_{7}$ in the form

$$
A(x, q t) B(x, t)=B(q x, t) A(x, t)
$$

by the compatibility of the deformation equation and the original linear $q$ difference equation.

We take $A(x, t)$ to be of the form

$$
\begin{gathered}
A(x, t)=A_{0}(t)+x A_{1}(t)+x^{2} A_{2}, \\
A_{2}=\left(\begin{array}{cc}
\kappa_{1} & 0 \\
0 & 0
\end{array}\right), \quad A_{0}(t) \text { has eigenvalues } \theta_{1} t, 0, \\
\operatorname{det} A(x, t)=\kappa_{1} \kappa_{2} x^{3} .
\end{gathered}
$$

The matrix $B(x, t)$ is a rational function of the form

$$
B(x, t)=\frac{1}{x}\left(x I+B_{0}(t)\right) .
$$

Define $y=y(t), z_{i}=z_{i}(t)(i=1,2)$ by

$$
A_{12}(y, t)=0, \quad A_{11}(y, t)=\kappa_{1} z_{1}, \quad A_{22}(y, t)=z_{2},
$$

so that

$$
z_{1} z_{2}=\kappa_{2} y^{3}
$$

The matrix $A(x, t)$ can be parametrized as

$$
A(x, t)=\left(\begin{array}{cc}
\kappa_{1}\left((x-y)(x-\alpha)+z_{1}\right) & w(x-y) \\
\kappa_{1} w^{-1}(\gamma x+\delta) & \kappa_{2}(x-y)+z_{2}
\end{array}\right) .
$$


Here

$$
\begin{aligned}
\alpha & =\frac{1}{\kappa_{1}}\left[y^{-1}\left(\theta_{1} t-\kappa_{1} z_{1}-z_{2}\right)+\kappa_{2}\right], \\
\gamma & =z_{2}-\kappa_{2}(2 y+\alpha) \\
\delta & =-y^{-1}\left(\alpha y+z_{1}\right)\left(\beta y+z_{2}\right) .
\end{aligned}
$$

Introduce $z$ by

$$
z_{1}=\frac{y^{2}}{\kappa_{1} q z}, \quad z_{2}=\kappa_{1} \kappa_{2} q y z
$$

Then the matrix $B_{0}(t)=\left(B_{i j}\right)$ is parametrized as follows:

$$
\begin{aligned}
B_{11} & =-q \bar{z}\left(\kappa_{2}-\frac{y}{\bar{z}}\right) \\
B_{12} & =q w \bar{z} \\
B_{21} & =\frac{\kappa_{1} q \bar{z}}{w\left(1-\kappa_{1} q \bar{z}\right)}\left(-\bar{\alpha}-\frac{\bar{y}}{\kappa_{1} q \bar{z}}\right)\left(\kappa_{2}-\frac{y}{\bar{z}}\right), \\
B_{22} & =\frac{-\kappa_{1} q \bar{z}}{1-\kappa_{1} q \bar{z}}\left(-\bar{\alpha}-\frac{\bar{y}}{\kappa_{1} q \bar{z}}\right) .
\end{aligned}
$$

Set further

$$
b_{1}=\frac{1}{\theta_{1}}, \quad b_{2}=-\frac{\theta_{1}}{\kappa_{1} \kappa_{2}}, \quad b_{3}=\frac{1}{\kappa_{1} q}, \quad a_{4}=-\kappa_{2} .
$$

Equation (2.143) are equivalent to

$$
\begin{aligned}
\frac{y \bar{y}}{a_{4}} & =\frac{b_{2} t \bar{z}}{\bar{z}-b_{3}}, \\
\frac{z \bar{z}}{b_{3}} & =-\frac{y}{a_{4}}, \\
\frac{\bar{w}}{w} & =-\frac{\bar{z}}{b_{3}}+1 .
\end{aligned}
$$

We have a constraint

$$
\frac{b_{1} b_{2}}{b_{3}}=q \frac{1}{a_{4}}
$$

$q-P\left(A_{7}\right)$ is (2.160) and (2.161).

\subsection{Lax form of $q-P\left(A_{7}^{\prime}\right)$}

Consider a $2 \times 2$ matrix system with polynomial coefficients

$$
Y(q x, t)=A(x, t) Y(x, t) .
$$


We express the deformation equation

$$
Y(x, q t)=B(x, t) Y(x, t)
$$

and can express the $q$-Painlevé equation of type $A_{7}^{\prime}$ in the form

$$
A(x, q t) B(x, t)=B(q x, t) A(x, t)
$$

by the compatibility of the deformation equation and the original linear $q$ difference equation.

We take $A(x, t)$ to be of the form

$$
\begin{gathered}
A(x, t)=A_{0}(t)+x A_{1}(t)+x^{2} A_{2}, \\
A_{2}=\left(\begin{array}{cc}
\kappa_{1} & 0 \\
0 & 0
\end{array}\right), \quad A_{0}(t) \text { has eigenvalues } \theta_{1} t, 0, \\
\operatorname{det} A(x, t)=\kappa_{1} \kappa_{2} x^{2} .
\end{gathered}
$$

The matrix $B(x, t)$ is a rational function of the form

$$
B(x, t)=\frac{1}{x}\left(x I+B_{0}(t)\right) .
$$

Define $y=y(t), z_{i}=z_{i}(t)(i=1,2)$ by

$$
A_{12}(y, t)=0, \quad A_{11}(y, t)=\kappa_{1} z_{1}, \quad A_{22}(y, t)=z_{2},
$$

so that

$$
z_{1} z_{2}=\kappa_{2} y^{2}
$$

The matrix $A(x, t)$ can be parametrized as

$$
A(x, t)=\left(\begin{array}{cc}
\kappa_{1}\left((x-y)(x-\alpha)+z_{1}\right) & w(x-y) \\
\kappa_{1} w^{-1}(\gamma x+\delta) & z_{2}
\end{array}\right) .
$$

Here

$$
\begin{aligned}
\alpha & =\frac{1}{\kappa_{1}} y^{-1}\left(\theta_{1} t-\kappa_{1} z_{1}-z_{2}\right), \\
\gamma & =z_{2}+\kappa_{2}, \\
\delta & =-y^{-1} z_{2}\left(\alpha y+z_{1}\right) .
\end{aligned}
$$

Introduce $z$ by

$$
z_{1}=\frac{y^{2}}{\kappa_{1} q z}, \quad z_{2}=\kappa_{1} \kappa_{2} q z
$$


Then the matrix $B_{0}(t)=\left(B_{i j}\right)$ is parametrized as follows:

$$
\begin{aligned}
B_{11} & =q y, \\
B_{12} & =q w \bar{z}, \\
B_{21} & =\frac{-\kappa_{1} q y}{w\left(1-\kappa_{1} q \bar{z}\right)}\left(-\bar{\alpha}-\frac{\bar{y}}{\kappa_{1} q \bar{z}}\right), \\
B_{22} & =\frac{-\kappa_{1} q \bar{z}}{1-\kappa_{1} q \bar{z}}\left(-\bar{\alpha}-\frac{\bar{y}}{\kappa_{1} q \bar{z}}\right) .
\end{aligned}
$$

Set further

$$
b_{1}=\frac{1}{\theta_{1}}, \quad b_{2}=-\frac{\theta_{1}}{\kappa_{1} \kappa_{2}}, \quad b_{3}=\frac{1}{\kappa_{1} q}, \quad b_{4}=-\kappa_{2} .
$$

Equation (2.166) are equivalent to

$$
\begin{aligned}
\frac{y \bar{y}}{a_{4}} & =-\frac{\bar{z}\left(\bar{z}-b_{2} t\right)}{\bar{z}-b_{3}}, \\
\frac{z \bar{z}}{b_{3}} & =\frac{y^{2}}{a_{4}}, \\
\frac{\bar{w}}{w} & =-\frac{\bar{z}}{b_{3}}+1 .
\end{aligned}
$$

We have a constraint

$$
\frac{b_{1} b_{2}}{b_{3}}=q \frac{1}{a_{4}} .
$$

$q-P\left(A_{7}^{\prime}\right)$ is (2.183) and (2.184).

\section{Degenerations}

Some replacements of the parameters for the degenerations of $q$-Painlevé equations were given in the paper, [6], for example. In this section, we present the replacements of the parameters of the Lax formalisms.

Replace in $q-P\left(A_{3}\right), t$ by $\varepsilon t, y$ by $\varepsilon y, z$ by $\varepsilon z, a_{3}$ by $\varepsilon a_{3}, a_{4}$ by $\varepsilon^{-1} a_{4} b_{3}$ by $\varepsilon b_{3}$ and $b_{4}$ by $\varepsilon^{-1}$ and let $\varepsilon$ tend to zero. Then we obtain $q-P\left(A_{4}\right)$ :

$$
\begin{gathered}
\frac{y \bar{y}}{a_{3} a_{4}}=-\frac{\left(\bar{z}-b_{1} t\right)\left(\bar{z}-b_{2} t\right)}{\bar{z}-b_{3}}, \\
\frac{z \bar{z}}{b_{3}}=-\frac{\left(y-a_{1} t\right)\left(y-a_{2} t\right)}{a_{4}\left(y-a_{3}\right)} . \\
\frac{b_{1} b_{2}}{b_{3}}=q \frac{a_{1} a_{2}}{a_{3} a_{4}}
\end{gathered}
$$


For the sake of simplification of notation, the replacement and the succeeding limiting process will be written as follows:

$$
\begin{gathered}
t \rightarrow \varepsilon t \quad y \rightarrow \varepsilon y, \quad z \rightarrow \varepsilon z, \\
a_{3} \rightarrow \varepsilon a_{3}, \quad a_{4} \rightarrow \varepsilon^{-1} a_{4} \quad b_{3} \rightarrow \varepsilon b_{3}, \quad b_{4} \rightarrow \varepsilon^{-1} .
\end{gathered}
$$

By the use of notation as above, the degeneration from the Lax form of $q-P\left(A_{3}\right)$ to that of $q-P\left(A_{4}\right)$ is given by the following scheme:

$q-P\left(A_{3}\right)$ to $q-P\left(A_{4}\right)$ :

$$
\begin{gathered}
t \rightarrow \varepsilon t \quad y \rightarrow \varepsilon y, \quad z \rightarrow \varepsilon z, \\
a_{3} \rightarrow \varepsilon a_{3}, \quad a_{4} \rightarrow \varepsilon^{-1} a_{4} \quad b_{3} \rightarrow \varepsilon b_{3}, \quad b_{4} \rightarrow \varepsilon^{-1}, \\
x \rightarrow \varepsilon x, \quad z_{1} \rightarrow \varepsilon^{2} z_{1}, \quad w \rightarrow \varepsilon^{-1} w, \quad \kappa_{1} \rightarrow \varepsilon^{-1} \kappa_{1}, \quad \kappa_{2} \rightarrow \varepsilon, \\
\alpha \rightarrow \varepsilon \alpha, \quad \beta \rightarrow \varepsilon^{-1} \beta, \quad \delta \rightarrow \varepsilon \delta, \\
Y(x, t) \rightarrow x^{\log _{q} \varepsilon} Y(x, t), \quad A(x, t) \rightarrow \varepsilon A(x, t), \\
A_{0}(t) \rightarrow \varepsilon A_{0}(t), \quad A_{2} \rightarrow \varepsilon^{-1} A_{2}, \quad B_{0}(t) \rightarrow \varepsilon B_{0}(t), \\
B_{11} \rightarrow \varepsilon B_{11}, \quad B_{12} \rightarrow \varepsilon B_{12}, \quad B_{21} \rightarrow \varepsilon B_{21}, \quad B_{22} \rightarrow \varepsilon B_{22} .
\end{gathered}
$$

$q-P\left(A_{4}\right)$ to $q-P\left(A_{5}\right)$ :

$$
a_{3} \rightarrow \varepsilon, \quad b_{2} \rightarrow \varepsilon^{-1} b_{2}, \quad \theta_{2} \rightarrow \varepsilon
$$

$q-P\left(A_{4}\right)$ to $q-P\left(A_{5}\right)^{\sharp}$ :

$$
\begin{gathered}
t \rightarrow \varepsilon t, \quad a_{1} \rightarrow \varepsilon^{-1} a_{1}, \quad a_{2} \rightarrow \varepsilon, \quad b_{1} \rightarrow \varepsilon b_{1}, \quad b_{2} \rightarrow \varepsilon^{-1} b_{2}, \\
\theta_{1} \rightarrow \varepsilon^{-1} \theta_{1}, \quad \theta_{2} \rightarrow \varepsilon .
\end{gathered}
$$

$q-P\left(A_{5}\right)$ to $q-P\left(A_{6}\right)$ :

$t \rightarrow \varepsilon t, \quad a_{1} \rightarrow \varepsilon^{-1} a_{1}, \quad a_{2} \rightarrow \varepsilon, \quad b_{1} \rightarrow \varepsilon b_{1}, \quad b_{2} \rightarrow \varepsilon^{-1} b_{2}, \quad \theta_{1} \rightarrow \varepsilon^{-1} \theta_{1}$.

$q-P\left(A_{5}\right)^{\sharp}$ to $q-P\left(A_{6}\right)$ :

$$
a_{3} \rightarrow \varepsilon, \quad b_{2} \rightarrow \varepsilon^{-1} b_{2}
$$

$q-P\left(A_{5}\right)^{\sharp}$ to $q-P\left(A_{6}\right)^{\sharp}$ :

$$
a_{1} \rightarrow \varepsilon, \quad b_{1} \rightarrow \varepsilon b_{1} .
$$

$q-P\left(A_{6}\right)$ to $q-P\left(A_{7}\right)$ :

$$
a_{1} \rightarrow \varepsilon, \quad b_{1} \rightarrow \varepsilon b_{1} .
$$

$q-P\left(A_{6}\right)^{\sharp}$ to $q-P\left(A_{7}\right)$ :

$$
a_{3} \rightarrow \varepsilon, \quad b_{2} \rightarrow \varepsilon^{-1} b_{2} .
$$

$q-P\left(A_{6}\right)^{\sharp}$ to $q-P\left(A_{7}^{\prime}\right)$ :

$$
a_{3} \rightarrow \varepsilon^{-1}, \quad a_{4} \rightarrow \varepsilon a_{4}, \quad \kappa_{2} \rightarrow \varepsilon \kappa_{2} .
$$




\section{Degeneration from $q-P\left(A_{2}\right)$ to $q-P\left(A_{3}\right)$}

The Lax form of $q-P\left(A_{2}\right)$ was given in the Sakai's paper, [8]. This Lax form yields the Lax form of $q-P\left(A_{3}\right)$ by a process of coalescence.

\subsection{Lax form of $q-P\left(A_{2}\right)$}

In this subsection, we illustrate the Lax form of $q-P\left(A_{2}\right)$ in the paper, [8].

Consider a $2 \times 2$ matrix system with polynomial coefficients

$$
Y(q x, t)=A(x, t) Y(x, t) .
$$

We express the deformation equation

$$
Y(x, q t)=B(x, t) Y(x, t)
$$

and can express the $q$-Painlevé equation of type $A_{2}$ in the form

$$
A(x, q t) B(x, t)=B(q x, t) A(x, t)
$$

by the compatibility of the deformation equation and the original linear $q$ difference equation.

We take $A(x, t)$ to be of the form

$$
\begin{gathered}
A(x, t)=A_{0}(t)+x A_{1}(t)+x^{2} A_{2}(t)+x^{3} A_{3}, \\
A_{3}=\left(\begin{array}{cc}
\kappa_{1} & 0 \\
0 & \kappa_{2}
\end{array}\right), \quad A_{0}(t) \text { has eigenvalues } \theta_{1} t, \theta_{2} t, \\
\operatorname{det} A(x, t)=\kappa_{1} \kappa_{2}\left(x-a_{1}\right)\left(x-a_{2}\right)\left(x-a_{3}\right)\left(x-a_{4}\right)\left(x-a_{5} t\right)\left(x-a_{6} t\right) .
\end{gathered}
$$

We have

$$
\kappa_{1} \kappa_{2} a_{1} a_{2} a_{3} a_{4} a_{5} a_{6}=\theta_{1} \theta_{2} .
$$

The matrix $B(x, t)$ is a rational function of the form

$$
B(x, t)=\frac{x}{\left(x-a_{5} q t\right)\left(x-a_{6} q t\right)}\left(x I+B_{0}(t)\right) .
$$

Define $\lambda=\lambda(t), \mu=\mu(t)$ and $\tilde{\mu}=\tilde{\mu}(t)$ by

$$
A_{12}(\lambda, t)=0, \quad A_{11}(\lambda, t)=\kappa_{1} \tilde{\mu}, \quad A_{22}(\lambda, t)=\kappa_{2} \mu,
$$

so that

$$
\mu \tilde{\mu}=\kappa_{1} \kappa_{2}\left(\lambda-a_{1}\right)\left(\lambda-a_{2}\right)\left(\lambda-a_{3}\right)\left(\lambda-a_{4}\right)\left(\lambda-a_{5} t\right)\left(\lambda-a_{6} t\right) .
$$


The matrix $A(x, t)$ can be parametrized as

$$
A(x, t)=\left(\begin{array}{cc}
\kappa_{1} W(x, t) & \kappa_{2} w L(x, t) \\
\kappa_{1} w^{-1} X(x, t) & \kappa_{2} Z(x, t)
\end{array}\right),
$$

Here

$$
\begin{aligned}
L(x, t) & =x-\lambda, \\
Z(x, t) & =(x-\lambda)\left(x^{2}+(\gamma+\lambda) x+\delta\right)+\mu \\
W(x, t) & =(x-\lambda)\left(x^{2}+\left(-\gamma+\lambda-\sigma_{1}\right) x+\tilde{\delta}\right)+\tilde{\mu} \\
X(x, t) & =\frac{W(x) Z(x)-\prod_{i=1}^{6}\left(x-a_{i}\right)}{L(x)}, \\
\delta & =\frac{1}{\kappa_{1}-\kappa_{2}}\left[\kappa_{1}\left(2 \lambda^{2}-\sigma_{1} \lambda+\sigma_{2}+\gamma\left(\gamma+\sigma_{1}\right)\right)-\frac{1}{\lambda}\left(\kappa_{1} \tilde{\mu}+\kappa_{2} \mu-\theta_{1}-\theta_{2}\right)\right] \\
\tilde{\delta} & =\frac{1}{\kappa_{1}-\kappa_{2}}\left[-\kappa_{2}\left(2 \lambda^{2}-\sigma_{1} \lambda+\sigma_{2}+\gamma\left(\gamma+\sigma_{1}\right)\right)+\frac{1}{\lambda}\left(\kappa_{1} \tilde{\mu}+\kappa_{2} \mu-\theta_{1}-\theta_{2}\right)\right] \\
\tilde{\mu} & =\frac{1}{\mu} \prod_{i=1}^{6}\left(\lambda-a_{i}\right), \quad \sigma_{1}=\sum_{i=1}^{6} a_{i}, \quad \sigma_{2}=\sum_{i<j} a_{i} a_{j} .
\end{aligned}
$$

If $q \kappa_{1}=\kappa_{2}$, then Equation (4.3) are equivalent to

$$
\begin{array}{r}
(\lambda-\underline{\nu})(\lambda-\nu)=\frac{\left(\lambda-a_{1}\right)\left(\lambda-a_{2}\right)\left(\lambda-a_{3}\right)\left(\lambda-a_{4}\right)}{\left(\lambda-a_{5} t\right)\left(\lambda-a_{6} t\right)} \\
\left(1-\frac{\nu}{\bar{\lambda}}\right)\left(1-\frac{\nu}{\lambda}\right)=\frac{a_{5} a_{6}}{q} \frac{\left(\nu-a_{1}\right)\left(\nu-a_{2}\right)\left(\nu-a_{3}\right)\left(\nu-a_{4}\right)}{\left(a_{5} a_{6} t+\theta_{1} / \kappa_{2}\right)\left(a_{5} a_{6} t+\theta_{2} / \kappa_{2}\right)} \\
a_{5} a_{6} t \lambda \bar{\lambda}\left(a_{1} ; a_{2}+a_{3}+a_{4}+\bar{\gamma}-\nu\right)\left(\left(a_{5}+a_{6}\right) t+\gamma+\nu\right) \\
+q\left(a_{5} a_{6} t \nu+\theta_{1} / \kappa_{2}\right)\left(a_{5} a_{6} t \nu+\theta_{2} / \kappa_{2}\right)=0 .
\end{array}
$$

$q-P\left(A_{2}\right)$ is (4.19) and (4.20).

\subsection{Degeneration}

In this subsection, we give replacements of the parameters for the degeneration. By the use of notation in the Section 3 , the degeneration from the Lax 
form of $q-P\left(A_{2}\right)$ to that of $q-P\left(A_{3}\right)$ is given by the following scheme:

$$
\begin{gathered}
\lambda \rightarrow \varepsilon y, \quad \nu \rightarrow \varepsilon^{-1} z, \\
a_{1} \rightarrow \varepsilon a_{3}, \quad a_{2} \rightarrow \varepsilon a_{4}, \quad a_{3} \rightarrow-\varepsilon^{-1}, \\
a_{4} \rightarrow-\varepsilon^{-1} q \kappa_{2}^{-1} \kappa_{1}, \quad a_{5} \rightarrow \varepsilon a_{1}, \quad a_{6} \rightarrow \varepsilon a_{2}, \\
x \rightarrow \varepsilon x, \quad \mu \rightarrow \varepsilon z_{2}, \quad \tilde{\mu} \rightarrow \varepsilon q \kappa_{2}{ }^{-1} \kappa_{1} z_{1}, \quad \kappa_{1} \rightarrow \varepsilon^{-1} q^{-1} \kappa_{2}, \quad \kappa_{2} \rightarrow \varepsilon^{-1} \kappa_{2}, \\
\gamma \rightarrow \varepsilon^{-1}+\varepsilon \gamma_{1}+O\left(\varepsilon^{2}\right), \\
\gamma_{1}=\frac{1}{\kappa_{1}-\kappa_{2}}\left[y^{-1}\left(\left(\theta_{1}+\theta_{2}\right) t-\kappa_{1} z_{1}-\kappa_{2} z_{2}\right)\right. \\
\left.-\kappa_{2}\left(\left(a_{1}+a_{2}\right) t+a_{3}+a_{4}\right)+y\left(\kappa_{1}+\kappa_{2}\right)\right] .
\end{gathered}
$$

\section{Concluding remarks}

By the limiting procedure, we derived Lax forms of $q$-Painlevé equations which are obtained from $q-P\left(A_{3}\right)$. The degeneration scheme from the Lax forms of $q-P\left(A_{2}\right)$ is also given. However Lax forms of $q-P\left(A_{0}^{*}\right)$ and $q-P\left(A_{1}\right)$ do not appear today, the full degeneration pattern cannot be presented. An interesting future problem remains to find the relation between the Lax pairs in the paper, [3], and our result.

\section{Acknowledgments}

The author expresses his sincere gratitude to Professor Hidetaka Sakai, who gave suggestions about ideas on this research.

\section{References}

[1] G. D. Birkhoff, The generalized Riemann problem for linear differential equations and the allied problems for linear difference and $q$-difference equations, Proc. Amer. Acad. Arts Sci. 49 (1913), 521-568.

[2] B. Grammticos, A. Ramani and V. G. Papageorgiou, Do integrable mappings have the Painlevé property?, Phys. Rev. Lett. 67 (1991), 18251828 .

[3] M. Hay, J. Hietarinta, N. Joshi and F. Nijhoff, A Lax pair for a lattice modified KdV equation, reductions to $q$-Painlevé equations and associated Lax pairs, J. Phys. A: Math. Theor. 40 (2007), F61-F73. 
[4] M. Jimbo and H. Sakai, A q-analog of the sixth Painlevé equation, Lett. Math. Phys. 38 (1996), 145-154.

[5] A. Ramani, B. Grammaticos and J. Hietarinta, Discrete versions of the Painlevé equations, Phys. Rev. Lett. 67 (1991), 1829-1832.

[6] A. Ramani, B. Grammaticos, T. Tamizhmani and K. M. Tamizhmani, Special function solutions of the discrete painlevé equations, Comput. Math. Appl. 42 (2001), 603-614.

[7] H. Sakai, A q-analog of the Garnier system, Funkcial. Ekvac. 48 (2005), 273-297.

[8] H. Sakai, Lax form of the $q$-Painlevé equation associated with the $A_{2}^{(1)}$ surface, J. Phys. A: Math. Gen. 39 (2006), 12203-12210.

[9] H. Sakai, Problem: discrete Painlevé equations and their Lax forms, RIMS Kôkyûroku Bessatsu B2 (2007), 195-208.

[10] H. Sakai, Rational surfaces associated with affine root systems and geometry of the Painlevé equations, Comm. Math. Phys. 220 (2001), 165229. 